\title{
Prospective Identification of Oligoclonal/Abnormal Band of the Same Immunoglobulin Type as the Malignant Clone by Differential Location of M-Spike and Oligoclonal Band
}

\author{
Shikhar G. Vyas ${ }^{\mathrm{a}}$, Gurmukh Singha, b
}

\begin{abstract}
Background: Serum and urine protein electrophoreses and immunofixation electrophoreses are the gold standards in diagnosing monoclonal gammopathy. Identification of oligoclonal bands in post-treatment patients has emerged as an important issue and recording the location of the malignant monoclonal peak may facilitate prospective identification of a new "monoclonal" spike as being distinct from the malignant peak.
\end{abstract}

Methods: We recorded the locations of monoclonal spikes in descriptive terms, such as being in the cathodal region, mid-gamma region, anodal region, and beta region. The location of monoclonal or restricted heterogeneity bands in subsequent protein electrophoreses was compared to the location of the original malignant spike.

Results: In a patient with plasma cell myeloma, the original monoclonal IgG kappa band was located at the anodal end of gamma region. Post-treatment, an IgG kappa band was noted in mid-gamma region and the primary malignant clone was not detectable by serum protein immunofixation electrophoresis (SIFE) in post-treatment sample. Even though the $\kappa / \lambda$ ratio remained abnormal, we were able to recognize stringent complete response by noting the different location of the new IgG kappa band as a benign regenerative process.

Conclusions: Recording the location of the malignant monoclonal spike facilitates the identification of post-treatment oligoclonal bands, prospectively. Recognizing the regenerative, benign, bands in posttransplant patients facilitates the determination of stringent complete response despite an abnormal $\kappa / \lambda$ ratio.

Keywords: Monoclonal gammopathies; M-peak location; Oligoclonal band; Stringent complete response; Autologous stem cell transplantation; Plasma cell myeloma

Manuscript submitted June 15, 2017, accepted June 29, 2017

aDepartment of Pathology, Medical College of Georgia at Augusta University, 1120, 15th Street BI 2008A, Augusta, GA 30912, USA

${ }^{b}$ Corresponding Author: Gurmukh Singh, Department of Pathology, Medical College of Georgia at Augusta University, 1120 15th Street, BI 2008A, Augusta, GA 30912, USA. Email: gurmukhsinghmdphd@yahoo.com

doi: https://doi.org/10.14740/jocmr3109w

\section{Introduction}

Malignant myeloma/plasma cell myelomas are monoclonal proliferations of terminally differentiated B lymphocytes and are generally associated with circulating monoclonal immunoglobulin [1]. IgG kappa is the most common immunoglobulin type in myelomas $[2,3]$. Serum protein electrophoresis (SPEP), serum protein immunofixation electrophoresis (SIFE) and corresponding urine protein analyses (UPEP and UIFE) are the gold standards for diagnosis of monoclonal gammopathy $[4,5]$. Bone marrow examination is usually carried out for definitive diagnosis. Serum free light chain assay (SFLCA) has been advocated as an adjunct but its usefulness has been questioned [3, 6, 7].

Treatment with newer chemotherapeutics, namely, bortezomib and thalidomide congeners, has improved survival [8, 9]. Myeloma patients often receive autologous stem cell transplants (ASCTs) following chemotherapy. About $60 \%$, and probably more, of the patients who receive ASCT develop an oligoclonal pattern or abnormal bands, following treatment [10-12]. The oligoclonal band(s) complicate the interpretation of SPEP/ SIFE and SFLCA. We have chosen to use the term oligoclonal band, even when only one band may be noted, and are not in favor of using the term "abnormal" band, as the proteins are not abnormal. IgG kappa oligoclonal bands are the usual prominent "abnormal" bands and IgG kappa is also the most common immunoglobulin type of myelomas. Thus, for proper identification of stringent complete response and to avoid unnecessary treatment, it is imperative that we distinguish an oligoclonal band as not a residual or recurrent tumor, even though it is of the same immunoglobulin type as the initial malignant clone [13].

As reported in the results of a retrospective study, recording the location of the original monoclonal peak facilitates the identification of oligoclonal bands that may appear in a different area of the SPEP/SIFE results [13]. We also reported the use of recording the location of the peak as a tool in ensuring specimen integrity, a form of delta check for specimen mislabeling [14].

Here we report the prospective identification of an oligoclonal band of the same immunoglobulin type as the primary malignant clone.

\section{Methods}

SPEP was performed by agarose gel electrophoresis using 


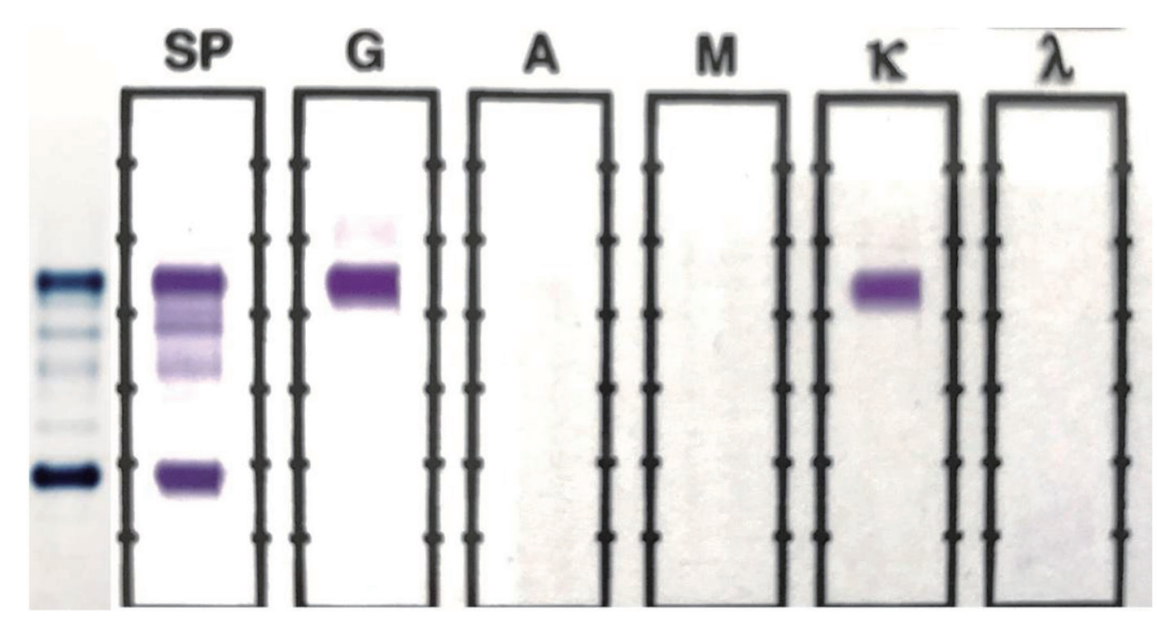

Figure 1. SPEP and SIFE in November 2016. A prominent monoclonal band is present at the beta-gamma interface on SPEP, which typed as IgG kappa on SIFE. The background immunoglobulins are markedly suppressed.

Helena SPIFE 3000 system. Quantitative evaluation for the usual proteins and any M-proteins was performed by scanning the gel at $595 \mathrm{~nm}$ on an EDC densitometer $[3,7,13]$. If the monoclonal protein overlapped a normal protein band, the concentration of the combined peak was reported. SFLCA was performed on Siemens ADVIA 2400 instrument, using Freelite kits and reagents from The Binding Site $[6,13]$.

A patient with known IgG kappa myeloma was treated with chemotherapy and ASCT. The diagnostic serum sample SPEP/SIFE result was recorded as showing an IgG kappa monoclonal spike at the anodal end of gamma region, partially overlapping the complement, or C3, band. A follow-up specimen 4 months post-ASCT showed a monoclonal IgG kappa band in the mid-gamma region, and was identified as being different from the primary malignant spike due to its location being different than that of the primary spike. The primary spike was no longer detectable in the specimen by SIFE. The information was reported in the electronic medical record, and the attending physician was notified of the interpretation by e-mail. A subsequent SPEP/SIFE, 2 months later, showed the same mid-gamma IgG kappa spike at a higher concentration, now with an additional IgG kappa band cathodal to the midgamma band.

In addition to the SPEP/SIFE results, the results for UPEP/ UIFE, SFLCA, immunoglobulin quantifications and bone marrow examination were evaluated at the three time points in the course of this patient.

\section{Results}

The patient, a 70-year-old male, was referred to our institution with a diagnosis of symptomatic multiple myeloma that evolved from MGUS, diagnosed 20 years earlier. At presentation to our institution, the findings included: a monoclonal IgG kappa spike of $2.19 \mathrm{~g} / \mathrm{dL}$, hemoglobin $11.5 \mathrm{~g} / \mathrm{dL}$, creatinine

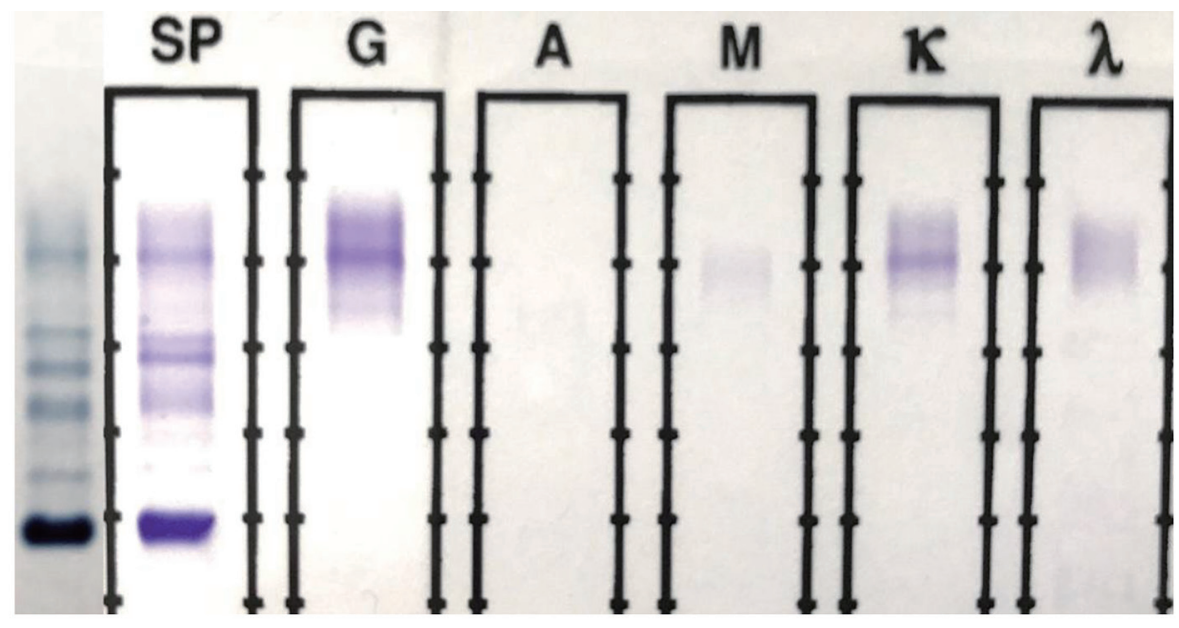

Figure 2. SPEP and SIFE in April 2017. The original malignant IgG kappa clone is absent on both SPEP and SIFE. A faint $(0.25$ $\mathrm{g} / \mathrm{dL}$ ) IgG kappa band is present in the mid-gamma region, clearly in a different location than the original malignant spike seen in Figure 1. Background immunoglobulins are more abundant than those in Figure 1. 


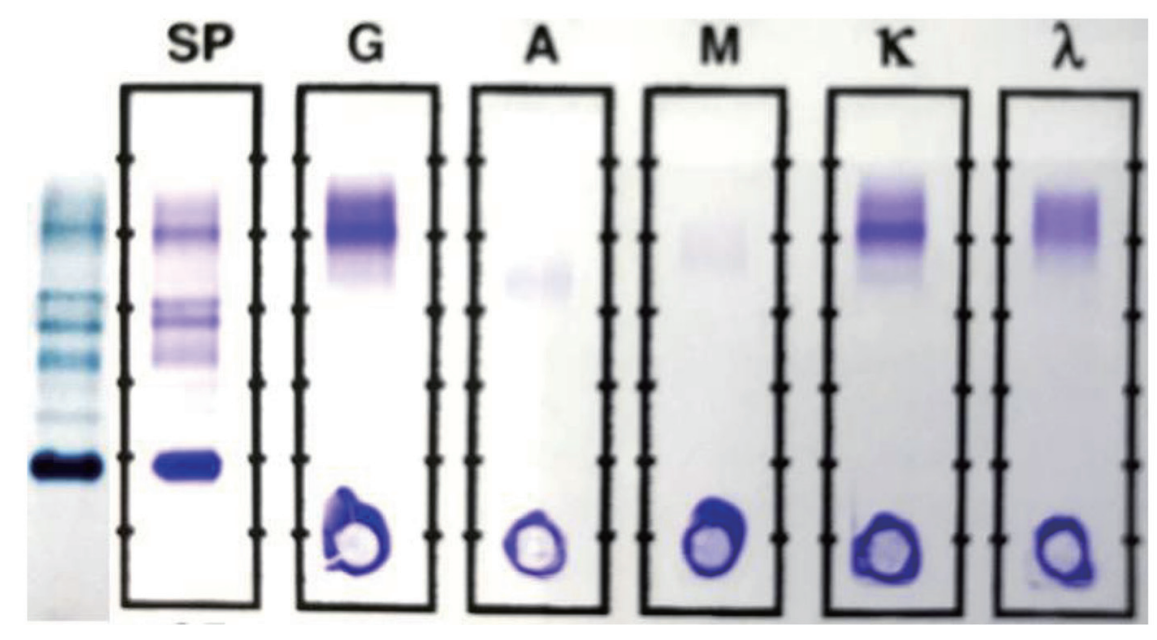

Figure 3. SPEP and SIFE in June 2017. The original malignant clone is still absent in both SPEP and SIFE. The mid-gamma IgG kappa band, first noted in April is present at a higher concentration $(0.38 \mathrm{~g} / \mathrm{dL})$ and an additional, cathodal IgG kappa band is noted at a concentration of about $0.12 \mathrm{~g} / \mathrm{dL}$. The second lower concentration band is more readily discernible in the densitometric scan, shown in Figure 4.

$1.23 \mathrm{mg} / \mathrm{dL}$, albumin $3.4 \mathrm{~g} / \mathrm{dL}$, calcium $8.1 \mathrm{mg} / \mathrm{dL}, \operatorname{IgG} 2,631$ $\mathrm{mg} / \mathrm{dL}, \operatorname{IgA} 25 \mathrm{mg} / \mathrm{dL}$, IgM $17 \mathrm{mg} / \mathrm{dL}$, serum free kappa chain $9.25 \mathrm{mg} / \mathrm{L}$, serum free lambda chain $0.71 \mathrm{mg} / \mathrm{L}$ and $\kappa / \lambda$ ratio of 13.03 . Bone marrow examination revealed $20 \%$ plasma cells with kappa chain restriction. Other illnesses included arthritis, type 2 diabetes mellitus, hypertension, hyperuricemia, hypothyroidism, with history of deep vein thrombosis and prostatic adenocarcinoma. Following chemotherapy, the patient received ASCT in December 2016, and follow-up SPEP/SIFE and other tests were done in April 2017 and June 2017.

The original SPEP/SIFE results are shown in Figure 1. A prominent IgG kappa band at the anodal end of gamma region was noted, partially overlapping the $\mathrm{C} 3$ band. The post-ASCT specimen SPEP/SIFE results are shown in Figures 2 and 3. In April 2017, no evidence of original malignant clone in the anodic gamma regions was discernible on electrophoreses. A low level IgG kappa band $(0.25 \mathrm{~g} / \mathrm{dL})$ was obvious in the midgamma region.

The SPEP/SIFE results in June 2017 revealed the midgamma IgG kappa band at a higher concentration. In addition, a new cathodal IgG kappa band was also identified (Figs. 3 and 4). These findings suggest a good response to treatment with oligoclonal bands of the same immunoglobulin type as the original malignant clone, but in clearly different locations than the anodic malignant clone. The results of the SPEP/ SIFE, UPEP/UIFE, serum free light chains, and immunoglobulin levels are shown in Table 1.

\section{Discussion}

New chemotherapeutics have improved survival of plasma cell myeloma patients $[8,9]$. Following chemotherapy, many patients are administered ASCTs. Despite prolongation of progression-free survival, however, the positive impact of ASCT in improving overall survival is controversial and actual sur- vival may not be improved by ASCT [10-20].

ASCT often induces the development of oligoclonal bands, and the latter may interfere with the recognition of a recurrent malignant immunoglobulin spike. In the search for a cure, recognition of minimal residual disease is important as implied by the persistence or recurrence of a malignant immunoglobulin protein. Since almost all patients relapse despite chemotherapy and ASCT, it could be assumed that almost all patients have undetected minimal residual disease, and the lack of detectable monoclonal spike on SPEP/SIFE does not exclude residual disease [20]. Various methods, including flow cytometry of bone marrow, liquid biopsy of peripheral blood plasma cells, molecular analysis of bone marrow cells, and mass spectrometric analysis of immunoglobulins, are being used or investigated to ascertain residual or minimal residual disease $[16,21]$.

In this report, we emphasize that recording the location of the malignant immunoglobulin peak facilitated prospective identification of a non-malignant oligoclonal band. Such restricted heterogeneity bands are common in post-ASCT patients [13]. Recognizing the location of the new "band" as being different from that of the malignant spike, despite the oligoclonal band being of the same immunoglobulin type as the initial malignant clone, allows one to determine it as representing regenerative, benign immunoglobulin rather than recurrence or persistence of a malignant clone. The development of a second oligoclonal band, further cathodal to the mid-gamma band provides additional supports to our hypothesis that the mid-gamma band is a response to the bone marrow reconstitution following ASCT. The increase in background IgG levels without any evidence of recurrence of the malignant clone further supports this argument. This is further supported by the return of the $\kappa / \lambda$ ratio to neutral or normal range in the third sample.

While the location of the new mid-gamma band, in a different location than the initial malignant clone, is strongly suggestive of a benign oligoclonal response, it does not rule 


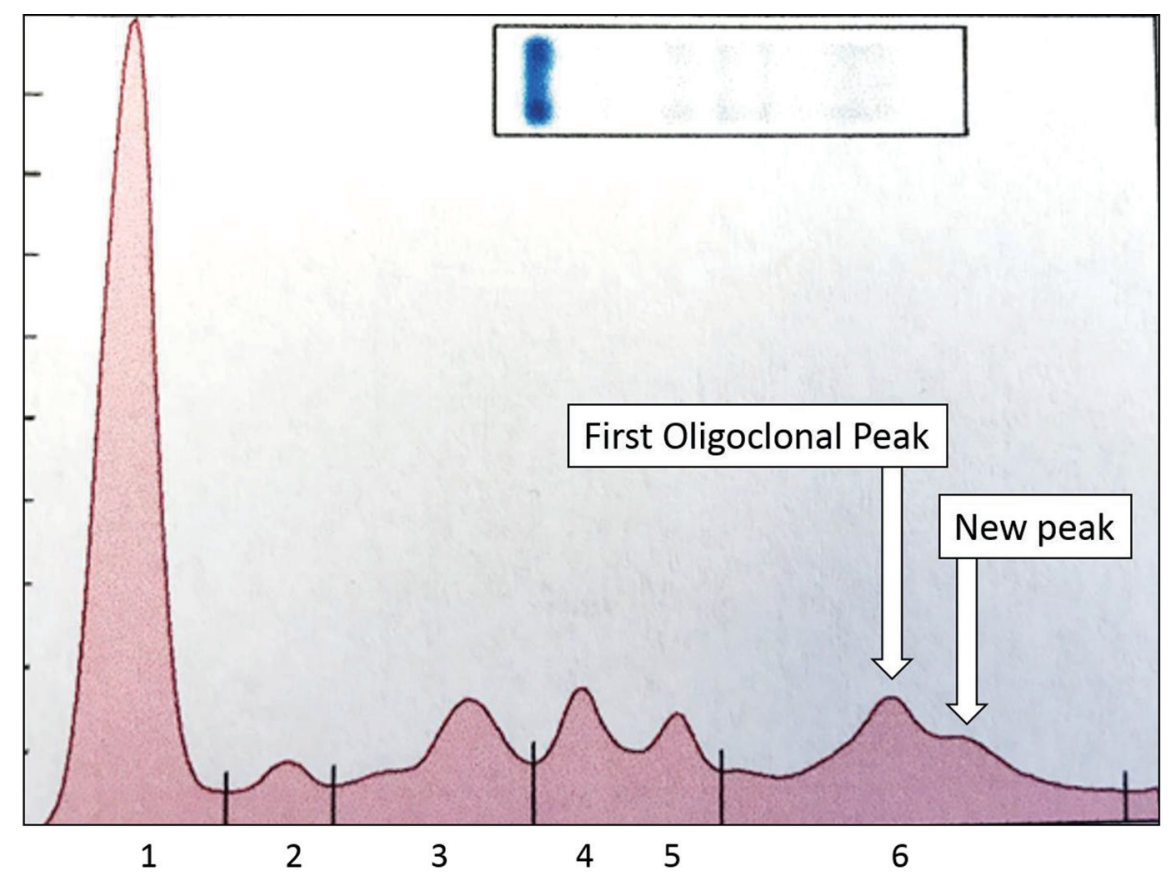

Figure 4. Densitometric scan of SPEP from Figure 3 shows the two spikes in gamma region, both of which typed as IgG kappa and constituted about 0.38 and $0.12 \mathrm{~g} / \mathrm{dL}$. The higher concentration peak is more anodal to the lower concentration one. The other, normal, peaks are: 1 = albumin; 2 = alpha 1 globulin; $3=$ alpha 2 globulin; 4 = beta 1 globulin; $5=$ beta 2 globulin; $6=$ gamma globulins.

out the development of a second malignant clone. Definitive recognition of this band as an oligoclonal band would require disappearance of this band on subsequent samples. However, the development of a second band, further cathodal to the first, mid-gamma oligoclonal band supports its prospective identification as a normal regenerative response, and not a second or third malignant clone.

The results of SFLCAs warrant a comment. In November 2016, with a more than $2.0 \mathrm{~g}$ monoclonal IgG kappa, the $\kappa / \lambda$ ratio was kappa dominant as expected. Despite the lack of a detectable monoclonal spike and lack of residual myeloma in the bone marrow, the $\kappa / \lambda$ ratio remained kappa dominant in April 2017. The abnormal $\kappa / \lambda$ ratio was in all likelihood due to the IgG kappa oligoclonal band skewing the ratio in favor of kappa. The SFLCA result in the June 2017 sample being in normal range further supports the interpretation of the April SFLCA as being "false positive" $\kappa / \lambda$ ratio.

As we have documented earlier, $\kappa / \lambda$ ratio is an unreliable indicator of monoclonal gammopathy [3, 7]. An abnormal $\kappa / \lambda$ ratio is not diagnostic of monoclonal gammopathy, and a normal ratio does not exclude monoclonal gammopathy. Moreover, there appears to be a systematic under-estimation of lambda light chains by, at least, The Binding Site assay [3]. We propose that an abnormal $\kappa / \lambda$ ratio should not preclude the determination of stringent complete response, if all other parameters are met, as the abnormal ratio may be due to a benign, regenerative oligoclonal band of the same immunoglobulin type as the malignant clone. Recording the location of the monoclonal band, and comparing the locations of the malignant spike and a putative oligoclonal band should be carried out systematically.

\section{Conclusions}

1) Recording the location of the malignant monoclonal immunoglobulin spike may allow prospective recognition of benign,

Table 1. SPEP/SIFE Data Over Three Tests

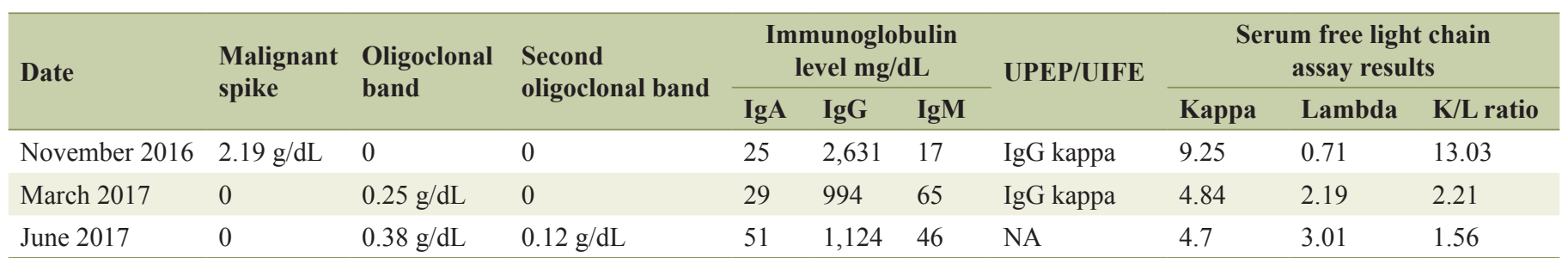

The initial malignant clone disappeared, while an oligoclonal pattern developed, in the following two SPEPs. A second oligoclonal band is also noted on the third SPEP. The $\mathrm{k} / \mathrm{\lambda}$ ratio is kappa dominant in the first two samples, but is in the normal range in the June 2017 sample. 
regenerative, oligoclonal bands of the same immunoglobulin type as the malignant clone.

2) An abnormal $\kappa / \lambda$ ratio, per se, should not exclude a stringent complete response in a patient with ASCT who may have an oligoclonal response that may be skewing the $\kappa / \lambda$ ratio, without implying the existence of malignant immunoglobulin.

\section{Acknowledgments}

The authors are grateful to Ms. Kathy Graham and, Ms. Renee Arthur, and Ms. Constance Johnson for technical assistance.

\section{References}

1. Kyle RA, Rajkumar SV. Multiple myeloma. Blood. 2008;111(6):2962-2972.

2. Osterborg A, Mellstedt H. Monoclonal and biclonal immunoglobulin-producing disorders. Eur J Haematol Suppl. 1989;51:11-18.

3. Singh G. Serum free light chain assay and kappa/lambda ratio: performance in patients with monoclonal gammopathy-high false negative rate for kappa/lambda ratio. J Clin Med Res. 2017;9(1):46-57.

4. Dimopoulos M, Kyle R, Fermand JP, Rajkumar SV, San Miguel J, Chanan-Khan A, Ludwig H, et al. Consensus recommendations for standard investigative workup: report of the International Myeloma Workshop Consensus Panel 3. Blood. 2011;117(18):4701-4705.

5. Kumar S, Paiva B, Anderson KC, Durie B, Landgren O, Moreau P, Munshi N, et al. International Myeloma Working Group consensus criteria for response and minimal residual disease assessment in multiple myeloma. Lancet Oncol. 2016;17(8):e328-346.

6. Bradwell A. Serum Free Light Chain Analysis plus Hevylite, 7 Ed. Birmingham, UK: The Binding Site Group Ltd. 2015.

7. Singh G. Serum free light chain assay and kappa/lambda ratio performance in patients without monoclonal gammopathies: high false-positive rate. Am J Clin Pathol. 2016;146(2):207-214.

8. Rajkumar SV, Kumar S. Multiple myeloma: diagnosis and treatment. Mayo Clin Proc. 2016;91(1):101-119.

9. Landgren O, Rajkumar SV. New developments in diagnosis, prognosis, and assessment of response in multiple myeloma. Clin Cancer Res. 2016;22(22):5428-5433.

10. Hall SL, Tate J, Gill D, Mollee P. Significance of abnormal protein bands in patients with multiple myeloma following autologous stem cell transplantation. Clin Biochem Rev. 2009;30(3):113-118.
11. Jimenez-Zepeda VH, Reece DE, Trudel S, Franke N, Winter A, Chen C, Tiedemann R, et al. Oligoclonal and monoclonal bands after single autologous stem cell transplant in patients with multiple myeloma: impact on overall survival and progression-free survival. Leuk Lymphoma. 2014;55(10):2284-2289.

12. Tovar N, Fernandez de Larrea C, Pedrosa F, Arostegui JI, Cibeira MT, Rosinol L, Elena M, et al. Differential humoral responses against heat-shock proteins after autologous stem cell transplantation in multiple myeloma. Ann Hematol. 2014;93(1):107-111.

13. Singh G. Oligoclonal pattern/abnormal protein bands in post-treatment plasma cell myeloma patients: implications for protein electrophoresis and serum free light chain assay results. J Clin Med Res. 2017;9(8):671-679.

14. Vyas S, Singh G. Location of monoclonal peak as a tool in checking specimen integrity. Prac Lab Med. 2017:4951.

15. Tovar N, de Larrea CF, Arostegui JI, Cibeira MT, Rosinol L, Rovira M, Elena M, et al. Natural history and prognostic impact of oligoclonal humoral response in patients with multiple myeloma after autologous stem cell transplantation: long-term results from a single institution. Haematologica. 2013;98(7):1142-1146.

16. Paiva B, van Dongen JJ, Orfao A. New criteria for response assessment: role of minimal residual disease in multiple myeloma. Blood. 2015;125(20):3059-3068.

17. de Larrea CF, Cibeira MT, Elena M, Arostegui JI, Rosinol L, Rovira M, Filella X, et al. Abnormal serum free light chain ratio in patients with multiple myeloma in complete remission has strong association with the presence of oligoclonal bands: implications for stringent complete remission definition. Blood. 2009;114(24):4954-4956.

18. Jo JC, Yoon DH, Kim S, Lee K, Kang EH, Jang S, Park $\mathrm{CJ}$, et al. Clinical significance of the appearance of abnormal protein band in patients with multiple myeloma. Ann Hematol. 2014;93(3):463-469.

19. Fujisawa M, Seike K, Fukumoto K, Suehara Y, Fukaya M, Sugihara H, Takeuchi M, et al. oligoclonal bands in patients with multiple myeloma: its emergence per se could not be translated to improved survival. Cancer Sci. 2014;105(11):1442-1446.

20. Attal M, Lauwers-Cances V, Hulin C, Leleu X, Caillot D, Escoffre M, Arnulf B, et al. Lenalidomide, bortezomib, and dexamethasone with transplantation for myeloma. $\mathrm{N}$ Engl J Med. 2017;376(14):1311-1320.

21. Mills JR, Kohlhagen MC, Dasari S, Vanderboom PM, Kyle RA, Katzmann JA, Willrich MA, et al. Comprehensive assessment of M-proteins using nanobody enrichment coupled to MALDI-TOF mass spectrometry. Clin Chem. 2016;62(10):1334-1344. 